\title{
Freehand mm-wave imaging with a compact MIMO radar
}

\author{
Guillermo Álvarez-Narciandi, Jaime Laviada and Fernando Las-Heras,Senior Member, IEEE
}

\begin{abstract}
This paper presents a freehand imaging system, which takes full-advantage of a compact multiple-input multipleoutput (MIMO) radar to provide real-time results by means of freehand movements. The system is based on a commercial radar-on-chip, equipped with four receivers and two transmitters, and an optical tracking system (i.e., motion capture system) for retrieving its position and attitude. The reduced size of the radar enables its handheld operation, making possible to create a synthetic aperture by moving the scanner over the area of interest. Due to the new degrees of freedom with respect to previous implementations based on quasi-monostatic topologies, a novel calibration strategy for the system is required in order to mitigate positioning errors. After calibration, simulations and measurements are accomplished revealing that the novel freehand multistatic setup significantly outperforms the monostatic setup in terms of scanning speed and image quality. Multimedia material is provided to illustrate this performance.
\end{abstract}

Index Terms-SAR imaging; mm-wave imaging; FMCW radar; MIMO imaging; multistatic imaging; real-time imaging; freehand scanner; motion capture.

\section{INTRODUCTION}

$\mathbf{E}$ LECTROMAGNETIC imaging systems are able to provide camera-like images with some penetration capabilities, enabling the inspection of the inner layers of targets [1]. These systems are used, under different implementations, in multiple applications such as nondestructive evaluation [2], [3], medicine [4] or security [5], [6], [7].

Among the multiple imaging systems, those based on synthetic aperture radar (SAR) are widespread when performing qualitative imaging [1]. The lateral resolution of these systems is proportional to the size of the aperture, whereas the range resolution depends on the bandwidth. As a consequence, traditionally, these systems rely on a dense set of transmitters and receivers [5], whose number can be reduced resorting to multiple-input multiple-output (MIMO) (also known as multistatic) schemes [8]. Alternatively, other systems use the movement of targets to apply inverse SAR techniques [9].

In general, the previous systems require relatively bulky hardware and, for some applications, compactness is an interesting feature. In this regard, a microwave camera of reduced size operating between 20 and $30 \mathrm{GHz}$, offering a tradeoff between size and resolution, has been presented [10]. Moreover, it has been shown that multiview approaches can be used to increase both the image quality and volume under test [11], [12], [13], [14]. A different microwave camera of reduced size based on chaotic excitation SAR, which has low

This work has been supported by the Ministerio de Educación y Formación Profesional of Spain under the FPU grant FPU15/06431, by the Ministerio de Ciencia, Innovación y Universidades under project RTI2018-095825-B-I00 and by the Principado de Asturias/FEDER under project IDI/2018/000191.

The authors are with the Department of Electrical Engineering, University of Oviedo, Gijón, 33203, Spain (e-mail: alvareznguillermo@uniovi.es).

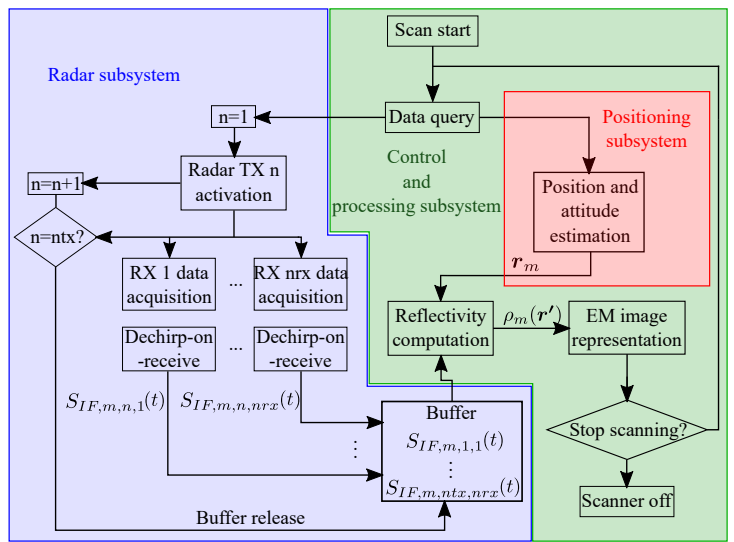

Figure 1. Workflow of the scanning system.

resolution but can be used in applications where high-fidelity images are not necessary, has been presented in [15].

In this context, the development of mm-wave technology has opened the possibility of using very compact on-chip systems [16], [17] at the expense of low resolution due to their small aperture. However, modern advances in position tracking [18], [19] have enable the use of this kind of compact radars together with freehand movements in order to generate much larger synthetic apertures [20]. Despite this major breakthrough, the system was relatively slow due to the use of a quasi-monostatic setup. In this paper, the previous system is extended to a MIMO configuration and a calibration technique, which mitigates positioning errors, is proposed. This entails a significantly faster scanning speed and higher-quality images.

\section{MultistaCtiC FREEHAND MM-WAVE IMAGING}

\section{A. System architecture}

The imaging system allows retrieving real-time highresolution images moving the scanner by hand. The architecture of this scanning system was presented in [20] for a quasi-monostatic configuration, but a brief description of the three subsystems of the scanner is given below:

- Control and processing subsystem: formed by a conventional PC or laptop, sets up the scanning system and communicates with the other subsystems. In addition, it computes the reflectance of the volume under scan and displays the radar images in real-time (see Fig. 1). To deal with the nonuniform nature of the acquired data, as the scanner is moved by hand, a strategy to balance the sample density is implemented in this subsystem. In particular, the area over the volume under scan is discretized in voxels shaped as cuboids of size $b \times b \times h$ inside of which only a limited number of acquisitions, $Q$, is allowed. Hence, after $Q$ acquisitions in a given cuboid are performed, other acquisitions in the same cuboid will 
be discarded. The parameter $b$ together with the value of $Q$, regulate the density of samples, whilst the parameter $h$ (height of the cuboids) provides the necessary flexibility with respect to a canonical surface (e.g., a plane) to the operator of the system, so handheld scanning can be performed. While a lower value of $h$ results in higher quality images, it also entails an increase of the scanning time since many samples would be discarded. Hence, the height $h$ establishes a trade-off between image quality and acquisition speed [20]. Moreover, to limit the maximum displacement during an acquisition, the position of the radar is queried before and after the acquisition and, if the difference is higher than $1 \mathrm{~mm}$, the sample is discarded. The Doppler shift, limited as the radar moves parallel to the volume under scan, is also effectively reduced by this.

- Radar subsystem: composed of a mm-wave FMCW radaron-chip module BGT60TR24B by Infineon ${ }^{\circledR}$ [16]. The frequency waveform of the signal transmitted by the radar has an up-chirp saw tooth pattern centered at $f_{c}=60 \mathrm{GHz}$ with a bandwidth of $B W=6 \mathrm{GHz}$ and a chirp duration of $T_{s}=512 \mu \mathrm{s}$. The radar module has two transmitting antennas that are sequentially activated and four independent receivers, which perform dechirpon-receive operations concurrently (See Fig. 1). IF signals are sent to the control subsystem for further processing.

- Positioning subsystem: comprises an optical tracking system that retrieves the position and attitude of the radar. In particular, the motion capture system of Optitrack ${ }^{\circledR}$ [21], which can be deployed and calibrated in a few minutes, has been used. To track the radar several reflective markers are attached to its enclosure. These markers define a rigid body whose attitude and position are monitored.

\section{B. Imaging technique}

From the point of view of the imaging technique, the presented system faces two major challenges in contrast to conventional SAR imaging. First, the scanner is freely moved by hand and, consequently, measurements are not expected to be uniformly distributed as it is usually the case in standard synthetic aperture radar (SAR) techniques for either monostatic [5] or multistatic setups [8], [6]. Second, it is convenient to perform an on-the-fly update of the reflectance of the volume under test to provide a visual feedback to the operator moving the compact radar. Taking into account the previous considerations, a MIMO delay-and-sum (DAS) algorithm, adapted to FMCW radar signals, is used in this work. The formulation of this technique is described in [20] for a quasimonostatic configuration. Here, some changes are considered to use a multistatic configuration with $n t x$ transmitters and nrx receivers.

The complex form of the signal received by the $p$-th $\mathrm{RX}$ and transmitted by the $n$ - $t h$ TX at the $m$-th position, $\boldsymbol{r}_{m}$, assuming a point target and omitting amplitude variations, is given by

$$
\begin{aligned}
s_{r x, m, p, n}(t) & =s_{t x, m, n}\left(t-\tau_{m, p, n}\right)= \\
& =e^{j 2 \pi\left(f_{c}\left(t-\tau_{m, p, n}\right)+\frac{B W}{2 T_{s}}\left(t-\tau_{m, p, n}\right)^{2}\right)},
\end{aligned}
$$

where $\tau_{m, p, n}$ is the propagation delay, which is proportional to the distance from the $n$-th transmitter at the $m$-th radar position

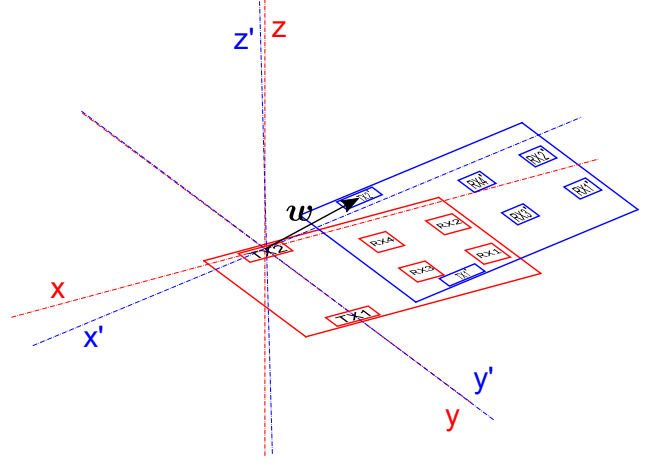

Figure 2. Scheme of the ideal case in which the rigid body and the radar module are aligned and $\boldsymbol{w}=(0,0,0)$ (red) and scheme of a case in which $\boldsymbol{w} \neq 0$ and the rigid body and the radar module are not aligned (blue).

to the point target, $R_{m, n}$, and from the point target to the $p$-th receiver at the $m$-th radar position, $R_{m, p}$. Therefore, the signal transmitted by the $n$-th transmitter and dechirped by the $p$-th receiver when the radar module is at position $\boldsymbol{r}_{m}$ is given by:

$$
s_{I F, m, p, n}(t)=e^{j 2 \pi\left(f_{b} t+f_{c} \tau_{m, p, n}-\frac{B W}{2 T_{s}} \tau_{m, p, n}^{2}\right)},
$$

where the beat frequency is $f_{b}=\frac{B W}{T_{s}} \tau_{m, p, n}$. It should be noted that, using a multistatic configuration, for each position $\boldsymbol{r}_{m}$ of the scanner, a set of $n t x \times n r x$ radar acquisitions will be performed and, thus, $n t x \times n r x$ IF signals will be retrieved:

$$
s_{I F, m}(t)=\left[s_{I F, m, 1,1}(t) \ldots s_{I F, m, n r x, n t x}(t)\right] .
$$

For distributed targets both the received and the IF signals can be expressed as a superposition of (1) and (2), respectively.

The reflectance, $\rho$, which yields the final electromagnetic image, is computed at each of the observation points, $\boldsymbol{r}^{\prime}$, of the grid in which the volume under test is discretized. For this purpose, the contributions of each radar measurement must be coherently combined. Moreover, new acquisitions can also be added to the result enabling the reflectance update during the scan. The first step to add coherently the different radar acquisitions is to compensate the extra phase term, which can be approximated, in case of close targets, by $f_{c} \tau_{m, p, n}$. After that, a range compression is done by making a Fourier transform in the time domain:

$$
s_{c, m, p, n}\left(\mathbf{r}^{\prime}, f\right)=\mathscr{F}\left\{s_{I F, m, p, n}(t) e^{-j 2 \pi f_{c} \tau_{m, p, n}}\right\},
$$

where $\mathscr{F}\{\cdot\}$ denotes the Fourier transform operator and $\tau_{m, p, n}=\frac{\left\|\mathbf{r}^{\prime}-\mathbf{r}_{m, p}\right\|_{2}+\left\|\mathbf{r}^{\prime}-\mathbf{r}_{m, n}\right\|_{2}}{c}=\frac{R_{m, p}+R_{m, n}}{c}$, where $\mathbf{r}_{m, p}$ and $\mathbf{r}_{m, n}$ are, respectively, the position of the $p$-th receiver and the $n$-th transmitter when the radar is at position $\boldsymbol{r}_{m}$. Similar to other standard time-domain techniques [22], [23], the used DAS algorithm can be expressed as

$$
\rho_{m}\left(\mathbf{r}^{\prime}\right)=\sum_{i=1}^{m} \sum_{p=1}^{n r x} \sum_{n=1}^{n t x} s_{c, i, p, n}\left(\mathbf{r}^{\prime}, \frac{B W}{T_{s}} \frac{R_{m, p}+R_{m, n}}{c}\right),
$$

which allows the use of nonuniformly spaced data at the expense of an efficiency reduction when compared to Fast Fourier Transform based methods [24], [6]. 




Figure 3. Scheme of the proposed setup to align the radar module with the rigid body. The picture also shows the basic layout of the radar antennas.

\section{Radar calibration}

In order to process the IF signal of each channel (i.e., transmitter-receiver pair) using (5), it is necessary to accurately know the position of each transmitter and receiver. Nonetheless, the optical tracking system only provides the position of the centroid of the (virtual) rigid body defined by (three or more) reflective markers attached to the radar, and its attitude. Although the relative positions between the radar antennas can be accurately found from the layout, the position and attitude offsets with respect to the rigid body are unknown. Moreover, direct estimations of these offsets by measuring the positions of the markers and the radar board can result in high inaccuracies. Consequently, a calibration stage, based on exploiting the radar properties, has been used to estimate the offset from a reference position of the radar layout to the centroid of the rigid body, $\boldsymbol{w}$, and the angular offset between the the rigid body and the radar board. It should be remarked that this calibration should be performed only once, when the reflective markers are attached to the radar module (in this paper, a 3D-printed enclosure was designed for this purpose).

A scheme of an ideal situation in which the offset between one of the transmitters, taken as a reference, and the centroid of the rigid body is zero, and the rigid body is aligned with the radar module is depicted in red in Fig. 2. However, this is not the case in a real situation (shown in blue in Fig. 2) and if the value of $\boldsymbol{w}$ is not correctly estimated and the rigid body and the radar board are not aligned, then errors will arise during the scanning process. These errors in the antenna position estimation will render the coherent sum of the imaging algorithm less effective, making the retrieved images noisier and, depending on the magnitude of the misalignment, reducing notably their quality.

The proposed calibration procedure entails two steps. The first step aims to find the angular offset of the radar module with respect to the rigid body defined by the markers of the tracking system. This is accomplished by taking advantage of the symmetric architecture of the transmitting and receiving antennas of the RF front-end, which are in-phase at $60 \mathrm{GHz}$ according to the manufacturer specifications. A basic scheme of the layout of the chip (rotated in front of a metal plate) is depicted in Fig. 3. As it can be seen, when the radar module is perfectly aligned with a metal plate (i.e., $\theta=0^{\circ}$ and $\psi=0^{\circ}$ ),

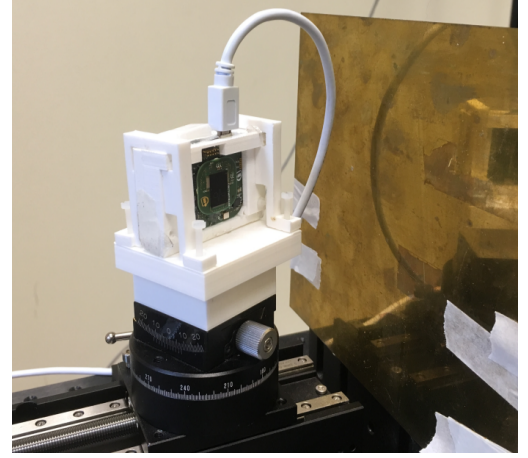

Figure 4. Setup used to align the radar module with the rigid body defined by the reflective markers.

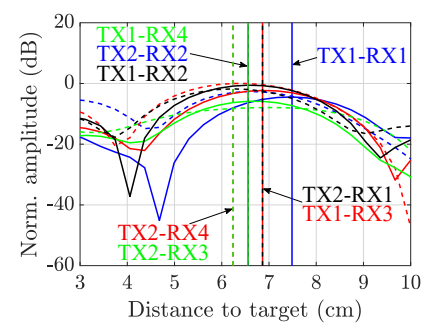

(a)

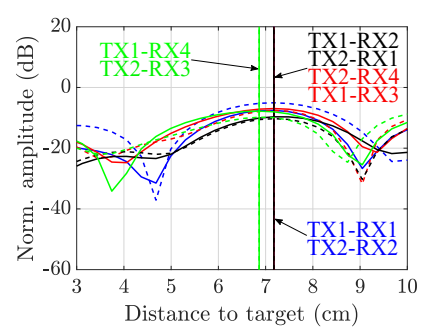

(b)
Figure 5. Dechirped signals of each TX-RX pair (a) before and (b) after the alignment. Symmetric TX-RX pairs, which should be in phase, share color.

the signal transmitted by TX1 and received by RX2 will be in-phase with the signal transmitted by TX2 and received by RX1. The same holds for other TX-RX pairs such as TX1RX4 and TX2-RX3 or TX1-RX1 and TX2-RX2. Thus, the radar module, with its 3D-printed plastic enclosure and the reflective markers, was mounted on a metric goniometer on top of a rotary stage diameter to accurately modify the pose of the radar in front of a metal plate until the signals corresponding to symmetric transmitter-receiver pairs are in-phase. Thus, the $\theta$ and $\psi$ offsets with respect to the plane defined by the metal plate, which is used as reference, can be found.

The complete calibration setup is shown in Fig. 4. To perform the calibration, the radar was programmed to perform continuous data acquisitions while the signals from each transmitter-receiver pair were monitored. Then, the pose of the radar was modified until the signals from the corresponding transmitter-receiver pairs were in phase. The dechirped signals before and after the calibration are shown in Fig. 5a and Fig. 5b, respectively. As it can be seen, none of previously mentioned transmitter-receiver pairs were in-phase before the calibration (vertical lines show the maximum of the IF signal retrieved by each receiver). On the other hand, after the calibration, all TX-RX pairs are in-phase. Finally, it is important to note that the previous approach does not provide information regarding rotations about the z-axis (Fig. 3). This offset was corrected by visual inspection since the impact of this offset, once the $\theta$ and $\psi$ offsets are corrected, is minor. It should be pointed out that, in order to systematize this task, a polarizer could be used to discriminate the correct rotations of the radar module around the z-axis. Finally, once the radar module is aligned, the pose of the rigid body is redefined in the motion capture software so that it matches the one of the radar module. 


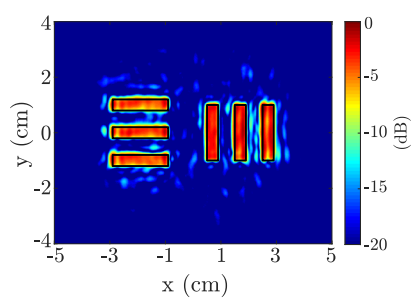

(a)

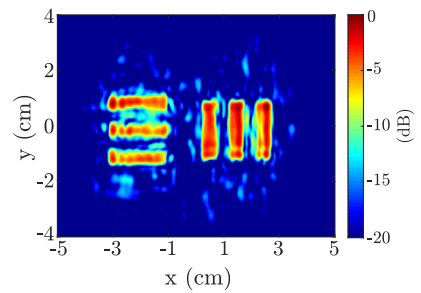

(c) (b)

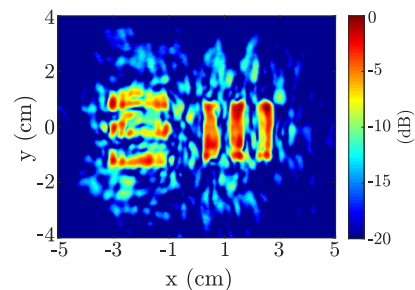

(d)

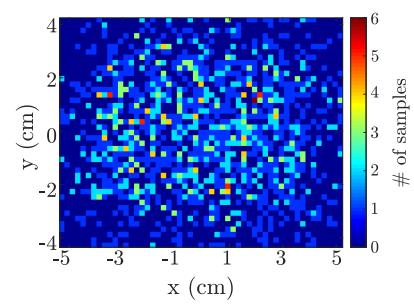

Figure 6. Reflectance image of the simulated target considering a radar module with 2 transmitters and 4 receivers for (a) $\boldsymbol{w}=(0,0,0) \mathrm{mm}$, for (c) $\boldsymbol{w}=(2,2,2) \mathrm{mm}$ and $\gamma_{\max }=10^{\circ}$, and for (d) $\boldsymbol{w}=(2,2,2) \mathrm{mm}$ and $\gamma_{\max }=15^{\circ}$. Acquisition positions are depicted in the heat map of (b).

The second step of the calibration process is to estimate the position offset, $\boldsymbol{w}$. For that purpose, an analogous strategy to the one followed in [20] for the quasi-monostatic case is followed. Thus, a target composed of several reflective markers on the same plane is scanned. Then, the electromagnetic image of the markers is systematically computed for different values of $\boldsymbol{w}$. The optimum value of $\boldsymbol{w}$ is obtained when the position of the markers in the electromagnetic image matches the position of the markers given by the optical tracking system and the maximum reflectance value corresponding to each marker is obtained at the vertical coordinate given by the tracking system (i.e., the image is focused at the vertical coordinate given by the tracking system). After that, this value is also corrected in the capture motion software so that, if the offset is perfectly estimated, $\boldsymbol{w}=(0,0,0)$.

Despite the previous systematic approach for calibration, some error is still expected in practice. The impact of this error is studied in the next section.

\section{NUMERICAL RESULTS}

To assess the impact of calibration errors and how they are mitigated by a correct procedure, several simulations were carried out. In particular, an ideal test target consisting of six metallic strips of size $20 \times 4 \mathrm{~mm}$ (three vertical and other three horizontal separated $6 \mathrm{~mm}$ ) was defined. The size of the cells was set to $2 \times 2 \times 20 \mathrm{~mm}$. The number of acquisitions per cell is shown in Fig. 6b. These positions were calculated by considering a regular 2D acquisition grid, which was modified by adding a random vector to each position and discarding the samples which were moved outside the cells. These random variations introduced to the initial regular grid emulate the nonuniform distribution of samples acquired during a manual scan. Regarding the radar model, the same layout as that of the commercial radar was used comprising $2 \mathrm{TX}$ and $4 \mathrm{RX}$.

The retrieved image considering a perfect alignment between the rigid body and the radar module and a zero offset, i.e., $\boldsymbol{w}=(0,0,0)$, is shown in Fig. 6a. As it can be observed, the six stripes are well-reconstructed and only little artifacts appear due to the nonunifom sampling. It is worth noting that the positioning errors for each transceiver of the radar module due to angular misalignment between the rigid body defined by the reflective markers and the radar module and the translation offset, $\boldsymbol{w}$, are given by

$$
\epsilon_{c a l, t}=\boldsymbol{R}(\varphi, \theta, \psi)\left[\boldsymbol{w}+\boldsymbol{R}_{e r r}\left(\varphi_{e r r}, \theta_{e r r}, \psi_{e r r}\right) \boldsymbol{r}_{t}-\boldsymbol{r}_{t}\right],
$$

where $\boldsymbol{R}(\varphi, \theta, \psi)$ is the rotation matrix obtained using the pose of the rigid body (yaw, pitch and roll angles) given by the optical tracking system, $\boldsymbol{r}_{t}$ is a vector containing the relative position of the transceiver $t$ with respect to a reference point of the radar module, which can be found from the radar layout, and $\boldsymbol{R}_{e r r}\left(\varphi_{\text {err }}, \theta_{\text {err }}, \psi_{\text {err }}\right)$ is the rotation matrix computed due to the angular misalignment: $\varphi_{e r r}, \theta_{e r r}$ and $\psi_{e r r}$. In a similar fashion to [20], eq. (6) shows that any calibration error (i.e., $\boldsymbol{w} \neq(0,0,0)$ or the existence of angular misalignment) results in a positioning error, which increases for high values of the attitude angles of the rigid body. Therefore, a strategy to mitigate calibration errors is to limit the attitude angles of the radar module while scanning by discarding samples in which any attitude angle is greater than a given threshold, $\gamma_{\max }$.

In order to illustrate the previous effect, the same test target was scanned for $\boldsymbol{w}=(2,2,2) \mathrm{mm}$ and two different attitude threshold values were considered: $\gamma_{\max }=10^{\circ}$ and $\gamma_{\max }=$ $15^{\circ}$. The attitude angles of the radar module at each sampling position were generated using a normal distribution. Results are depicted in Fig. 6c and Fig. 6d, respectively. As it can be observed, although the quality of the reconstructed target in the reflectance image for $\gamma_{\max }=10^{\circ}$ is degraded, the six stripes can be distinguished and their shape is well estimated. However, for $\gamma_{\max }=15^{\circ}$ a lot of artifacts on the image appear and the shape of the stripes is clearly distorted.

\section{MEAsurement RESUltS}

The enhancement on the image quality obtained as a result of the presented calibration process and the performance of the proposed MIMO system were assessed by measuring two sets of objects. First, a two euro coin, a two euro cent coin and a door key, arranged as depicted in Fig. 7a and covered by cardboard, were scanned. The size of the cuboids to balance the sample density was set to $1 \times 1 \times 20 \mathrm{~mm}$ and only one sample per cuboid was considered. In addition, the orientation angle threshold was set to $\gamma_{\max }=10^{\circ}$. The distribution of the 679 acquired samples, measured during $88 \mathrm{~s}$, is shown in Fig. $7 \mathrm{~b}$. The images obtained considering the data acquired using only 1 TX and $1 \mathrm{RX}$ without and with calibration are depicted in Fig. 7c and Fig. 7d, respectively. On the one hand, the image before calibration is clearly noisy and full of artifacts due to the poor coherency. Moreover, all the (fuzzy) objects in the uncalibrated image appear shifted with respect to their actual positions, depicted with white lines. On the other hand, the image after calibration clearly improves and the two euro coin can be inferred, but the resolution is still low due to the relatively low number of samples. 
The images retrieved using the 2 TXs and the 4 RXs of the radar module before and after the calibration are shown in Fig. 7e and Fig. 7f, respectively. Using this MIMO scheme, in both cases, despite using the same number of acquisitions as in the previous configuration, all the objects can be detected and all the energy is clearly more focused in the actual objects. However, without the calibration the objects appear shifted again and the energy is more spread, resulting in more artifacts than after the calibration. As it can be seen, in the image obtained after applying the calibration, the shape of all the objects is well-reconstructed, even the small hole of the handle of the door key. In order to quantify the image quality improvement when using the proposed calibration and when switching to a MIMO configuration, the target to clutter ratio (TCR) is used. The TCR is given by

$$
T C R=10 \log \left(\frac{N_{c} \sum_{(x, y) \in A_{t}}|\rho(x, y)|^{2}}{N_{t} \sum_{(x, y) \in A_{c}}|\rho(x, y)|^{2}}\right),
$$

where $A_{t}$ is the region corresponding to the target (i.e., the pixels where the target is located), $A_{c}$ is the rest of the image, $\rho(x, y)$ is the reflectance of pixel $(x, y)$ of the image, and $N_{t}$ and $N_{c}$ are the number of pixels of $A_{t}$ and $A_{c}$, respectively. The results are summarized in Table I. As discussed from a qualitative point of view, the maximum TCR is obtained for the image computed with the MIMO configuration after the calibration, used as reference to compute the image quality loss, denoted as $L$, of the other images.

Table I

TCR AND QUALITY LOSS OF THE RESULTS DISPLAYED IN FIG. 7.

\begin{tabular}{lccccc}
\hline & \multicolumn{2}{c}{1 TX 1 RX } & & \multicolumn{2}{c}{2 TX 4 RX } \\
\cline { 2 - 3 } \cline { 5 - 6 } & Pre-Cal & Post-Cal & & Pre-Cal & Post-Cal \\
\hline TCR $(d B)$ & 7.8 & 9.2 & & 12.4 & 14.3 \\
L $(d B)$ & 6.5 & 5.1 & & 1.9 & 0 \\
\hline
\end{tabular}

In conclusion, after the calibration the quality of the images is improved. Also, using a multistatic setup the scanning time is reduced, as more information is gathered for a fixed amount of samples. In a similar fashion, a scan of a given time yields higher-quality images using a multistatic setup.

In a second experiment, an in-depth study of the impact of the number of considered channels of the compact radar on the performance of the scanner was conducted. In this case, the system was tested by scanning the scissors shown in Fig. $8 \mathrm{~g}$ covered by cardboard. The size of the cuboids to balance the sample density was also set to $1 \times 1 \times 20 \mathrm{~mm}$, but the maximum number samples per cuboid was $P=2$. In addition, in order to mitigate positioning errors, the orientation angle threshold was also set to $\gamma_{\max }=10^{\circ}$. A total of 2640 measurements were acquired during $183 \mathrm{~s}$. The samples distribution is depicted in Fig. 8f. The obtained reflectance images are shown from Fig. $8 \mathrm{a}$ to Fig. 8e for the case of considering only $1 \mathrm{TX}$ and 1 RX, 1 TX and 2 RXs, 1 TX and 4 RXs, 2 TXs and 2 RXs, and all of the radar module transceivers (2 TXs and 4 RXs), respectively. In particular, it should be noted that, using all of the transceivers of the radar module, the blades and the handles of the scanned scissors can be clearly recognized. As it can

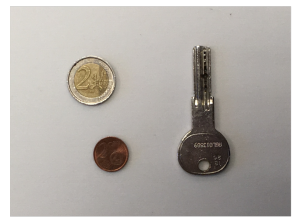

(a)

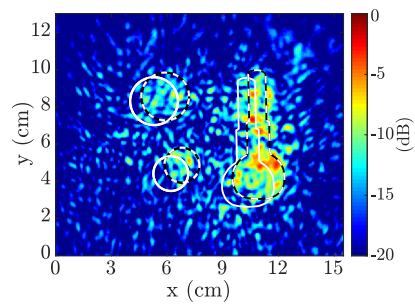

(c)

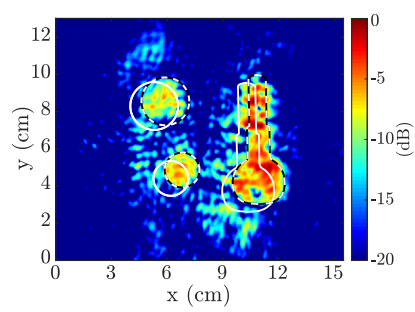

(e)

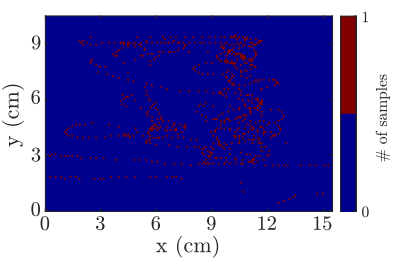

(b)

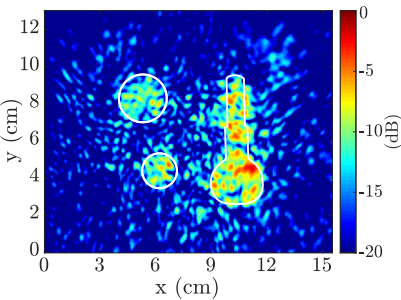

(d)

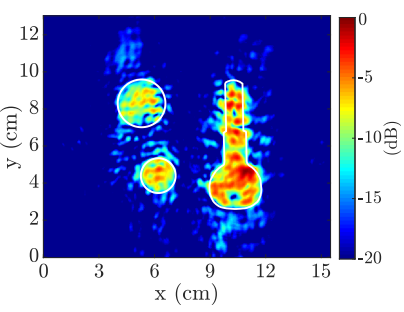

(f)
Figure 7. (a) Picture of the targets, (b) position of the acquisitions and image obtained using $1 \mathrm{TX}$ and $1 \mathrm{RX}$ (c) before, and (d) after calibration, and using the 2 TXs and the 4 RXs of the radar (e) before and (f) after calibration.

be observed, the quality of the retrieved image is significantly improved as more TXs and RXs are considered. As in the previous case, a quantitative analysis of these results using the TCR was performed. The obtained values, in the same line as the qualitative analysis, are summarized in Table II.

The videos of the real-time imaging results considering all the transceivers of the radar module for these tests, in which the evolution of the electromagnetic image as more data is acquired can be observed, are available in [25], [26].

\section{CONCLUSiON}

In this manuscript a freehand MIMO system with capacity to operate in real-time has been presented. The proposed system, which extends to a multistatic architecture the scanner presented in [20], comprises a mm-wave radar-on-chip module and an optical tracking system to compute its position and orientation while the operator of the scanner freely moves it over the area under scan with their hand. The system takes advantage of this movement of the radar to generate a synthetic aperture to obtain high-resolution images.

Table II

TCR AND QUALITY LOSS OF THE RESULTS DISPLAYED IN FIG. 8.

\begin{tabular}{lcccccc}
\hline & \multicolumn{3}{c}{$1 \mathrm{TX}$} & & \multicolumn{2}{c}{$2 \mathrm{TX}$} \\
\cline { 2 - 4 } \cline { 6 - 7 } & $1 \mathrm{RX}$ & $2 \mathrm{RX}$ & $4 \mathrm{RX}$ & & $2 \mathrm{RX}$ & $4 \mathrm{RX}$ \\
\hline TCR (dB) & 5.4 & 6.1 & 9.2 & & 8.3 & 10.6 \\
$\mathrm{~L}(\mathrm{~dB})$ & 5.2 & 4.5 & 1.4 & & 2.3 & 0 \\
\hline
\end{tabular}

The calibration of the system, in order to find the offset between the motion capture system and the radar system of 


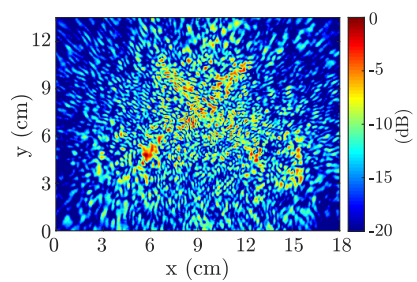

(a)

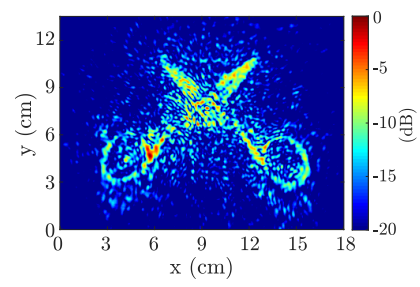

(c)

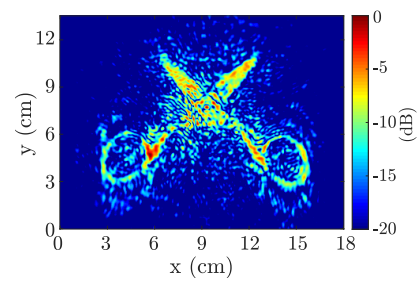

(e)

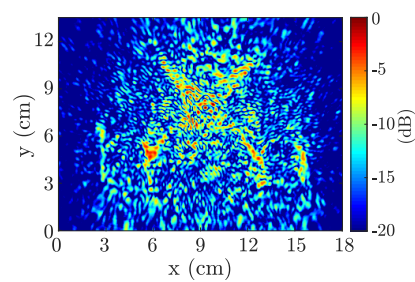

(b)

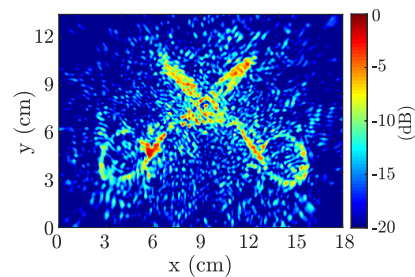

(d)

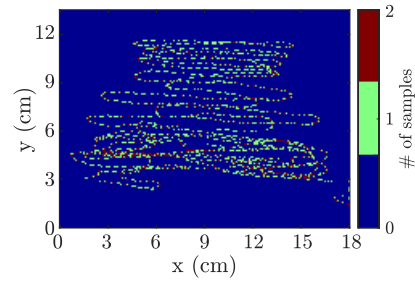

(f)

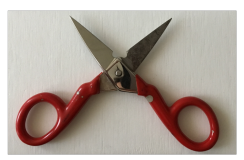

(g)

Figure 8. Computed reflectance image obtained using (a) $1 \mathrm{TX}$ and $1 \mathrm{RX}$, (b) $1 \mathrm{TX}$ and $2 \mathrm{RXs}$, (c) $1 \mathrm{TX}$ and 4 RXs, (d) 2 TXs and 2 RXs and (e) the 2 TXs and the 4 RXs of the radar module. (f) Heat map with the position of radar acquisitions and $(\mathrm{g})$ scanned scissors.

coordinates is critical to guarantee the good performance of the imaging algorithm. For this purpose, a relatively simple calibration method is proposed to find the attitude offset by rotating the radar in front of a metal plate until the received signals of symmetric channels are in-phase. In addition, the translation offset can be easily found by using as scanning target the markers of the optical tracking system in order to refer the radar system of coordinates and the motion capture system to the same reference. The impact of these calibration errors has been assessed by means of numerical simulations confirming a strong image quality degradation if the calibration is not properly accomplished.

Finally, the measurement results illustrate the performance of the multistatic system, showing that high-quality images can be achieved with a number of samples which would not be enough for a monostatic system. Thus, the proposed setup entails faster scanning speed and enhanced image quality.

\section{REFERENCES}

[1] M. Pastorino, Microwave Imaging. John Wiley \& Sons, Inc., 2010.

[2] S. Kharkovsky, J. T. Case, M. A. Abou-Khousa, R. Zoughi, and F. L. Hepburn, "Millimeter-wave detection of localized anomalies in the space shuttle external fuel tank insulating foam," IEEE Trans. Instrum. Meas., vol. 55, no. 4, pp. 1250-1257, Aug 2006.
[3] F. Boero, A. Fedeli, M. Lanini, M. Maffongelli, R. Monleone, M. Pastorino, A. Randazzo, A. Salvadè, and A. Sansalone, "Microwave tomography for the inspection of wood materials: Imaging system and experimental results," IEEE Trans. Microw. Theory Techn., vol. 66, no. 7, pp. 3497-3510, 2018.

[4] N. K. Nikolova, "Microwave imaging for breast cancer," IEEE Microwave Magazine, vol. 12, no. 7, pp. 78-94, Dec 2011.

[5] D. M. Sheen, D. L. McMakin, and T. E. Hall, "Three-dimensional millimeter-wave imaging for concealed weapon detection," IEEE Trans. Microw. Theory Techn., vol. 49, no. 9, pp. 1581-1592, Sep 2001.

[6] X. Zhuge and A. G. Yarovoy, "Three-dimensional near-field MIMO array imaging using range migration techniques," IEEE Trans. Image Process., vol. 21, no. 6, pp. 3026-3033, June 2012.

[7] J. Gao, Y. Qin, B. Deng, H. Wang, and X. Li, "Novel efficient 3D shortrange imaging algorithms for a scanning 1D-MIMO array," IEEE Trans. Image Process., vol. 27, no. 7, pp. 3631-3643, July 2018.

[8] S. S. Ahmed, A. Schiessl, and L. P. Schmidt, "A novel fully electronic active real-time imager based on a planar multistatic sparse array," IEEE Trans. Microw. Theory Techn., vol. 59, no. 12, pp. 3567-3576, Dec 2011.

[9] D. M. Sheen, R. T. Clark, J. Tedeschi, A. M. Jones, and T. E. Hall, "High-resolution 3D microwave imaging of a moving target using optical motion capture," in Passive and Active Millimeter-Wave Imaging XXII, D. A. Wikner and D. A. Robertson, Eds., vol. 10994, International Society for Optics and Photonics. SPIE, 2019, pp. $99-109$.

[10] M. T. Ghasr, M. A. Abou-Khousa, S. Kharkovsky, R. Zoughi, and D. Pommerenke, "Portable real-time microwave camera at $24 \mathrm{GHz}$," IEEE Trans. Antennas Propag., vol. 60, no. 2, pp. 1114-1125, Feb 2012.

[11] J. Laviada, A. Arboleya-Arboleya, Y. Alvarez, B. Gonzalez-Valdes, and F. Las-Heras, "Multiview three-dimensional reconstruction by millimetre-wave portable camera," Sci. Rep., vol. 7, Jul 2017.

[12] J. Laviada, A. Arboleya-Arboleya, and F. Las-Heras, "Multistatic millimeter-wave imaging by multiview portable camera," IEEE Access, vol. 5, pp. 19259-19268, 2017.

[13] J. Laviada, M. López-Portugués, A. Arboleya-Arboleya, and F. LasHeras, "Multiview mm-wave imaging with augmented depth camera information," IEEE Access, vol. 6, pp. 16869-16877, 2018.

[14] J. Laviada, M. T. Ghasr, M. López-Portugués, R. Zoughi, and F. LasHeras, "Multiview imaging with real-time microwave camera from known positions," in IEEE Antennas and Propagation Simposium 2018, Boston, USA, July 2018, pp. 1-3.

[15] M. J. Horst, M. T. Ghasr, and R. Zoughi, "A compact microwave camera based on chaotic excitation synthetic-aperture radar," IEEE Trans. Antennas Propag., vol. 67, no. 6, pp. 4148-4161, June 2019.

[16] I. Nasr, R. Jungmaier, A. Baheti, D. Noppeney, J. S. Bal, M. Wojnowski, E. Karagozler, H. Raja, J. Lien, I. Poupyrev, and S. Trotta, "A highly integrated $60 \mathrm{GHz}$ 6-channel transceiver with antenna in package for smart sensing and short-range communications," IEEE J. Solid-State Circuits, vol. 51, no. 9, pp. 2066-2076, Sept 2016.

[17] Texas Instruments. (2019, Sep.) $60 \mathrm{GHz}$ Radar-on-Chip. [Online]. Available: https://www.ti.com/tool/IWR6843AOPEVM

[18] H. He, P. Maheshwari, and D. J. Pommerenke, "The development of an EM-field probing system for manual near-field scanning," IEEE Trans. on Electromagn. Compat., vol. 58, no. 2, pp. 356-363, April 2016.

[19] C. Baer, J. Barowski, and I. Rolfes, "On the usability of low-cost inertial navigation systems for free-hand SAR imaging at GPR-frequencies," in 2017 IEEE Sensors Applications Symposium (SAS), March 2017.

[20] G. Álvarez-Narciandi, M. López-Portugués, F. Las-Heras, and J. Laviada, "Freehand, agile, and high-resolution imaging with compact mmwave radar," IEEE Access, vol. 7, pp. 95 516-95 526, 2019.

[21] OptiTrack. (2018, Feb.) Optitrack motion capture system. [Online]. Available: http://www.optitrack.com

[22] Z. Wang, J. Li, and R. Wu, "Time-delay- and time-reversal-based robust Capon beamformers for ultrasound imaging," IEEE Trans. Med. Imag., vol. 24, no. 10, pp. 1308-1322, Oct 2005.

[23] M. Klemm, I. J. Craddock, J. A. Leendertz, A. Preece, and R. Benjamin, "Improved delay-and-sum beamforming algorithm for breast cancer detection," International Journal of Antennas and Propagation, 2008.

[24] J. Yang, J. Thompson, X. Huang, T. Jin, and Z. Zhou, "FMCW radar near field three-dimensional imaging," in 2012 IEEE International Conference on Communications (ICC), June 2012, pp. 6353-6356.

[25] G. Álvarez Narciandi, J. Laviada, and F. Las-Heras. Freehand scan of hidden coins and key. University of Oviedo. [Online]. Available: https://youtu.be/PPwKsr3qciU

[26] $\ldots$. Freehand scan of hidden scissors. University of Oviedo. [Online]. Available: https://youtu.be/VRjjDHVeg4s 